EPJ Web of Conferences 58, 01019 (2013)

DOI: $10.1051 /$ epjconf/20135801019

(C) Owned by the authors, published by EDP Sciences, 2013

\title{
Speakable and unspeakable in physics of time
}

\author{
Enrico Prati ${ }^{1, a}$ \\ ${ }^{1}$ Laboratorio MDM, CNR-IMM, Via Olivetti 2, I-20864 Agrate Brianza, Italy
}

\begin{abstract}
In order to discriminate speakable and unspeakable in physics of time, the single ion optical atomic clock and the double time-slit experiments are considered. Their description clarifies that temporal quantities such as time and frequency are fundamentally unspeakable, while speakable is only related to ratios between wavelengths and to boundary conditions of stationary light beams. In addition, time intervals lower than the minimum period realized by the experimental apparatus are the results of an extrapolation out of the domain of definition of measurement operations. A toy model is employed to isolate the hidden source of self-recursivity in the definition of clocks and time, which resides in the abuse of language generated by terms such as duration and repetitive event, instead of interval extension and multiplicity. The timeless scenario which emerges from the experimental picture is theoretically supported by fundamental timelessness of the Hamiltonian formalism employed to define dynamics. Clock metrology, employed to parametrize dynamics in experiments, works successfully as it returns a discrete time variable, which approximates the parametrization of the trajectories in the phase space induced by the invariance of the action and of the time independent Hamiltonian.
\end{abstract}

\section{Introduction}

During the centuries, the developement of instruments for the measurement of time has reduced the limits of uncertainty from hours to small fractions of a second with the invention of atomic clocks. Scientists transferred the natural language about the notion of time into formal definitions and assumptions. In experiments, in GPS systems and to predict time evolution of systems with dynamics, the standard conceptual framework in which time is treated as a fundamental quantity is not critical. On the contrary, the inclusion of time among independent variables to provide an arena for Nature [1-5] is an inescapable question towards the comprehension of Nature at a fundamental level. Indeed, many key concepts employed in physics such as causality, locality, or the definition of a space-time background in some theories including some versions of quantum gravity [6] are based on time. In the following, it is shown that neither time is necessary to describe experiments apparently related to time physics, nor it is in the mathematical construction of dynamics. I describe two experiments apparently based on the concept of time in sections II and III, which are respectively the single $\mathrm{Hg}$ atom atomic clock [7] and the double time-slit experiment [10], in order to discriminate speakable and unspeakable. The counterintuitive result of the analysis is that time and frequency quantities are unspeakable, while the bare description of the experiments is based on wavelengths. With the help

\footnotetext{
ae-mail: enrico.prati@cnr.it
} 
of a toy model, in section IV, the hidden source of the self-recursivity of the definition of time and frequency is shown to be generated by time-related concepts introduced by the abuse of the natural language employed to qualify time, such as duration and repetitive events. Section V is dedicated to show that Hamiltonian dynamics can be defined without the use of time, and how the success of clock metrology comes from its ability to provide a good approximation of the natural parametrization of trajectories in phase space for which equations of motion look simple.

\section{Single ion optical atomic clock}

Thanks to their accuracy and stability, atomic clocks are the most precise instruments to define time intervals. Therefore, one might expect that a deep analysis of the most advanced atomic clock, for which the reference resonance is provided by the transition of a single atom, should clarify the nature of the observable under investigation. For consistency, here I refer to the specific implentation realized in 2001 at NIST with an $\mathrm{Hg}$ atom.[7] While the details can be found in the original work, here I explain in brief the working principle and I isolate the key points in the perpective of this analysis. Next, I show the issues connected to natural language to describe the experiment, and what is speakable about it. According to the standard view, like any good clock, the single ion clock has a device which produces periodic events (clock ticks), and a counter to accumulate and display. Being an atomic clock, it adds a third component: the resonance of a well isolated atomic transition. In other words, it is based on an optical transition at a much higher frequency, and a specialized frequency divider (frequency chain) is used (Figure 1). The apparatus consists of a self-referenced all-optical clock in which a femtosecond laser with repetition rate $f_{r}=1 \mathrm{GHz}$ is locked to the frequency of a single atom oscillator. The said femtosecond laser sends the beam into a microstructure fiber which operates a spectral broadening and produces a set of frequencies in the visible/near infrared. The low-frequency portion of the set is frequency-doubled and heterodyned against the high-frequency portion in a photodetector (PD1). In addition, the offset frequency $f_{0}$ common to all modes of the set is extracted. One specific frequency is heterodyned with the optical standard laser oscillator $\left(f_{H g}\right.$ $=532 \mathrm{THz}$ ), locked to the clock transition frequency of the single ${ }^{199} \mathrm{Hg}^{+}$ion, by the photodetector PD2, returning a beat frequency $f_{b}$. By employing two phase-locked loops (PLL) on $f_{0}$ and $f_{b}$, the spacing $f_{r}$ of the two is phase-locked to the $\mathrm{Hg}^{+}$standard. The output of the clock is the microwave domain frequency $f_{r}$, detected by the photodetector PD3, which is employed to generate the time standard. In this system, there are two implicit assumptions. One is that for a light beam $c=\lambda f$ where $c$ is a constant (which we call speed of light), while the second is that the locked frequencies are operated in stationary conditions. Therefore, let's investigate how this conversion from lengths to frequency is realized: the optical components such as the photodetectors and phase locked loops work thanks to interferometry in space: for instance the phase locked loop PLL2 changes the cavity length of the fs laser with a piezo mirror such that $f_{r}=a f_{0}$. While the description in terms of frequency is commonly adopted to express the final output as the duration of time intervals, the description of the mere facts about the clock has ground on employment of space boundary conditions. If one defines the bare experimental operations, the description of the atomic clock is based on an equipment which works in stationary conditions. The creation of a frequency chain is obtained by control of boundary conditions in space. The description of all the PLLs is done in terms of ratios between the wavelength of different light beams. Different frequencies may be next associated to photons of different wavelengths, by giving a meaning to the constant $c$ in terms of speed, which is a concept based on time, so it cannot contribute to its definition. So, if one rephrases the experiment in terms of the space boundary conditions of the stationary waves, there is technically no reason to employ an alternative set of definitions or concepts like time, time evolution and period, which would be 


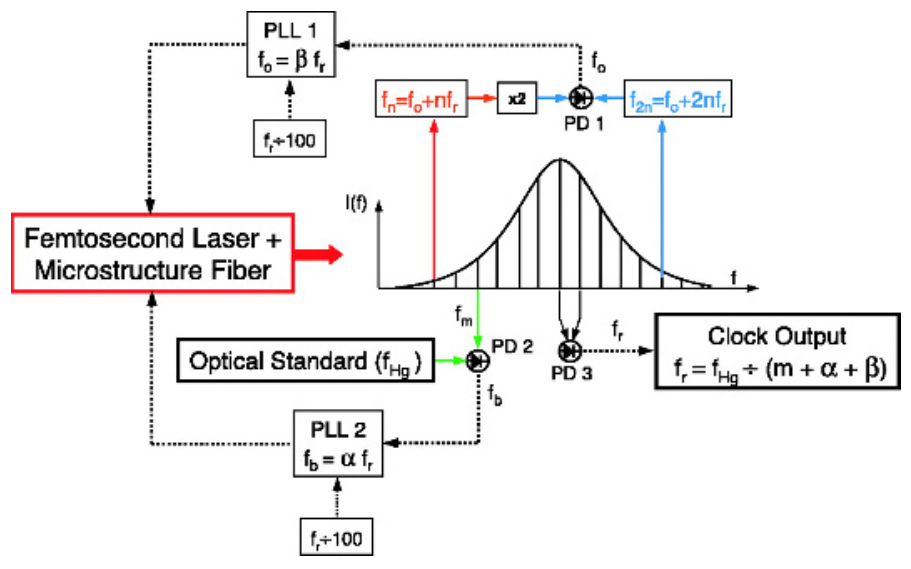

Figure 1. Single Hg ion optical atomic clock. From S.A. Diddams et al., An Optical Clock Based on a Single Trapped ${ }^{199} \mathrm{Hg}$ Ion, Science 293, 825 (2001). Reprinted with permission from AAAS.

possible if the concept of speed was available at this stage. Paradoxically, speakable on atomic clock experiment is ratios between wavelengths.

\section{Double time slit experiment}

While in the previous section the need to employ time to discuss atomic clock experiments in stationary conditions has been challenged, here the case of individual short time intervals in non stationary conditions is discussed. In the recent past, experiments have been reported about the so called double time slit setup.[10,11] This setup has been considered of great benefit for studying molecular dynamics at the attosecond time scale [12], by employing pulses which, because of their short duration, are said sub-cyclic. Intense pulses of radiation on the femtosecond scale show phenomena attributed to the opening of time slits for time intervals shorter than the period itself, at the attosecond scale. The authors of this field call their schemes as double-slit experiments operated in the time-energy domain. By following the standard phrasing for these experiments, the role of the slits is played by windows in a time of attosecond duration. Slits can be opened and closed by changing the temporal evolution of the field of a few cycle-laser pulse, so at any given time there is only a single electron in the doubleslit. To better understand the real phenomena described in these terms, here I explain in more details the experiment, according to Ref. [10]. Argon atoms are ionized by an intense few-cycle $760 \mathrm{~nm}$ laser pulses and the electrons are detected on two opposite detectors (Figure 2). The laser field is horizontally polarized, i.e., parallel to an axis defined by the electron detectors. The experimental observation consists of fringe pattern in the energy domain because time and energy are conjugated variables, attributed to the interference of temporally separated wave packets. By using phase-controlled fewcycle laser pulses, the temporal evolution of the field is manipulated, by gradually opening or closing the slits at different times. Therefore, depending on the field, one or two half-cycles - or anything in between - contribute to the electron amplitude for a given direction and electron energy. The experimenter shifts a glass wedge into the beam so the envelope of the pulse with respect of the carrier is delayed, achieving different conditions of self-interference. For the case of sin-like pulses, the effect is attributed to two slits with no which-way information in one direction of the emission (said positive, in the right direction $R$ ), and one slit with complete which-way information in the opposite direction $(L)$. 


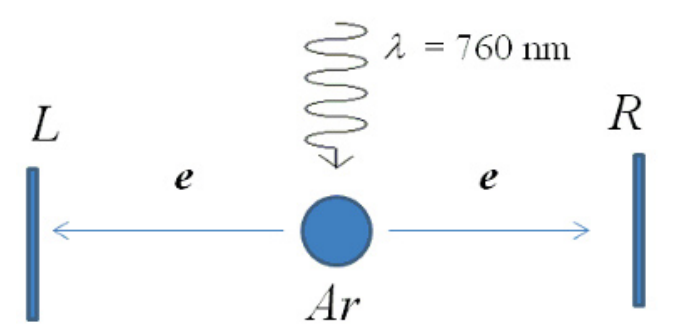

Figure 2. Double time slit apparatus. The left (L) and the right (R) screens collect the emission of electrons at the two opposite sides after the pulse of radiation hits the Ar atom.

Each argon atom emits at most one electron, which creates interference depending on the trajectories which contribute to its final state. The interpretation of this is obtained by looking at the conservation of the momentum in presence of the vector potential $A(t)$. As the momentum before the interaction of the electron bound to the atom is zero, then $\vec{p}-e \vec{A}\left(t_{0}\right)=0$, and the final result comes from the interference of all trajectories of momentum $\vec{p}$. Each slit is, according to the standard interpretation, a pair of slits. The time separation of these subslits depends on the electron energy, which is the quantity measured by the equipment. By inverting the energy-time relation, the experimental data provide a measurement of the time difference of the two subslits of about 500 as. In the simple's man model, the interpretation of the experiment is based on the assumption that the same electron is ionized twice in time. After the first time, the electron re-enters through the second slit and it re-exits.[13]. This view approximates the result of the path integral approach [14], for which all the paths contribute to the final result. A question arises about associating a time interval to this process below the time duration of a single period (or cycle). Does a temporal interpretation of the process below the period of a single cycle have something do to with the real process, if the equipment is unable to resolve such process? Time duration is defined in terms of counting of periods, so the employment of the concept of time interval here is out of the domain of definition. As the system provides a minimum period thanks to the laser frequency, the sub-cycle time slit separation is an extrapolation, whose value is calculated indirectly. Implicitly, one has extended the concept of time interval below the duration of a single cycle, but there is no direct measurement/evidence that some ordered time in the sense of the clock standard, which, as said, operates in stationary conditions thanks to space boundary conditions. The notion of time is defined by a clock apparatus, and the concept or duration of a narrow pulse below a single cycle is an arbitrary extrapolation of the same concept, with the further issue that it is not supported by any evidence when it is employed to interpret the experiment in terms of interval duration between time slits. While time duration is unspeakable as it consists only in a rephrasing of the same experiment (atomic clock) with other words (previous paragraph), time intervals of duration below a single cycle are unspeakable as they extend the previous (unspekable) concept out of the domain of definition.

\section{A toy experiment: the clack}

In order to better understand the origin of the inconsistency of the definition of time and frequency, and the abuse of natural language when we apply it in fundamental physics, here we introduce a toy system based on a fantasy atom syntetized by a civilization of an hypothetical planet. This system is based on ${ }^{666}$ frigerium and it is employed to define a new quantity, the quid. The operative definition 
of the quid is based on the description of the instrument to measure it: the clack. The clack is an instrument devoted to the measurement of quid intervals, which is measured in quix. By definition, one quix is a quid interval which consists of the duration of 1.000 .000 of quods of the radiation corresponding to the transition between two hyperfine levels of the ground state of the ${ }^{666}$ frigerium atom. In addition, to define the douq as the rate of occurence of a repetitive event. If $Q$ is the quod of a repetitive event, than the douq is the reciprocal $1 / Q$. To complete the model, the quod is a quid interval expressed in quixes. The first problem to a human is to provide some meaning to this set of definitions. After reading, no one gets any understanding of what is a clack and what it is going to measure in quixes. If one examines naively the definitions, he gets immediately that they consist of a set of mutually crossed definitions $P_{1}, P_{2}, P_{3}$ and $P_{4}$ :

1. quid $=P_{1}($ clack $)$

2. clack $=P_{2}($ quid,quix $)$

3. quix $=P_{3}($ quods, quid $)$

4. quod $=P_{4}$ (quix,quid)

The information provided by these definitions is apparently insufficient to capture their meaning. As we really want to understand their true meaning, we examine more deeply the other words implicitly used in the definitions. We may ask if the problem comes from the concept of repetitive event. We rule out the issue by saying that a repetitive event is an event which occurs with a multiplicity in quid. Next, we may ask if the problem comes from the concept of duration. We can easily recognize that the duration in quid is its extension, given by the number of standard quods in a quid interval. There is only one possible source of issues remaining, which is the only reference of something connected to our experience, which is the quod of the radiation. We may agree to experimentally obtain the quod of the radiation from $\lambda / k$ where $\lambda$ is the wavelength and $k$ is a constant in [m][quix] ${ }^{-1}$. This completes the picture, so now the definitions 1. and 2. are completed as follows:

3. quix $=P_{3}$ (quods, quid, quod of a radiation)

4. quod $=P_{4}$ (quix, quid)

5. quod of a radiation $=P_{5}$ (wavelength, quid, quix)

In other words, the toy model consists of a set of definition based on a reference atom and the concept of space. Excluding mass, radiation beams and space, there is no reason to suspect the existance of further independent quantities on that planet and to give any ontological status to the quid independently from the space. The problem for us, on Earth, is that this toy model considered totally useless on the hypothetical planet, it is not a toy. It coincides with the definition of clock (the clack), time (quid), second (quix) and period (quod), if only one replaces frigerium with cesium and $k$ with $c$ [15]. As said in Section II, at this stage the latter constant is not informative, as the notion of speed is implicitly related to that of time, which is under tentative construction. The toy formulation clarifies that, when we use natural language to talk about time, we replace the words of the attributes commonly adopted for all the other quantities with distinct words, connected to the concept of time: extension of an interval with duration, and multiplicity with respect to something with repetitive. In this sense, duration and repetitive are considered unspeakable, as they are used to define the same concept on which they are partially grounded. We may condense this argument by saying that there is no room in our definitions for the flow of quid. In the definition of time, clock, and period, an abuse of language 


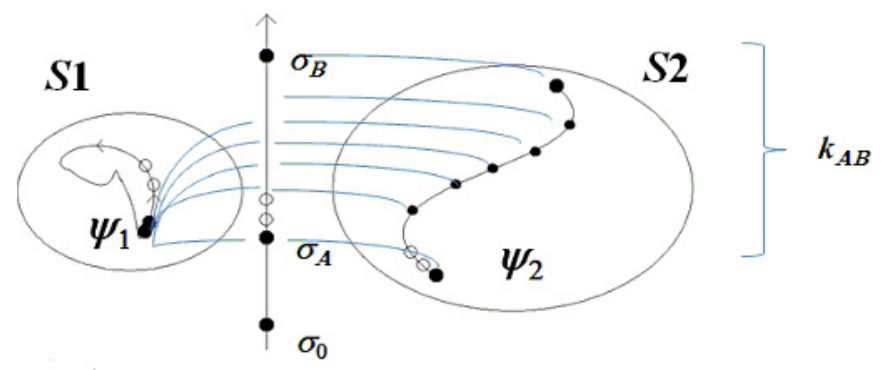

Figure 3. Generalized clock in phase space. The cyclic system $S 1$ allows to count $k_{A B}$ correpondences of the same state $\psi_{1}$ with different points of the phase space of $S 2$, thanks to the common parametrization $\sigma$ induced by the Hamiltonian constraint and the variational principle on the action. Under suitable stability conditions, $S 1$ acts as generalized clock for $S 2$, so motion described in terms of $\sigma$ is well approximated by motion as a function of the clock time $T=\alpha k_{A B}$ where $\alpha$ is a scaling factor.

based on the concept of duration and repetitivity erroneously supports the idea that time is an independent quantity, which refers to something real (or ontologically independent). If one reconsiders such definitions by replacing the time-related words with a more aseptic languange, there is no room to any sentence which has experimental ground on time and its flow.

\section{Timeless Hamiltonian formalism and generalized clocks}

Now, we turn to the role of time in hamiltonian mechanics. As already noted by Landau in a short note[16], there is in principle no need to attribute the meaning of time to the real parameter which parametrizes the Hamilton equations. In the recent past this idea has been extended to a formal derivation of the Hamilton equations, totally independent by the emplyoment of the concept of time (see [4] for the detailed discussion of this topic). Briefly, it is possible to consider trajectories in phase space and define a starting arbitrary parametrization, which, under suitable conditions and by imposing only the invariance of the time independent Hamiltonian along the trajectory together with a timeless action principle (Maupertuis principle), it returns a natural parametrization to express differentials of motion in a simple shape

$$
d \sigma=\left(\frac{\partial H}{\partial p_{i}}\right)^{-1} d q_{i}=-\left(\frac{\partial H}{\partial q_{i}}\right)^{-1} d p_{i}
$$

which differ from the Hamilton equations by the only fact that $\sigma$ does not represent the macroscopic metric time. $\sigma$ is a convenient parametrization of the system imposed by the energy conservation, so equations of motions are simple. In order to explain the reason why clocks are so useful to describe dynamics, we can separate a system $S$ in two subsystems $S 1$ and $S 2$. If one replaces the concept of periodicity in time (as explained in the previous section) with multiplicity in the phase space of a subsystem $S 1$ compared to a second subsystem $S 2$, it is consequently possible to use $S 1$ as generalized clock with respect to $S 2$. We may employ the multiplicity of cycles of $S 1$ in the phase space to associate an integer number to the corresponding trajectory of $S 2$ in the phase space. If $S 1$ operates under reasonable stability conditions, it discretely maps the parameter $\sigma$ of the Hamilton dynamics, so in the laboratory the equation of motion of $S 2$ appears well approximated by the Hamilton equations. Needless to say, any attempt to extend the properties of the cyclic subsystem employed as generalized 
clock below a single cycle is an attempt to apply concepts and methods out of their domain of definition. This view provides a rigorous approach to time in dynamics and the connection with clock experiments, totally consistent with speakable and unspeakable aspects related to the interpretation of the experiments described in the previous sections.

\section{Conclusion}

To conclude, time related experiments to isolate speakable and unspeakable in the physics of time have been discussed. The fundamental timelessness of Nature which arises from experiments finds a theoretical support in Hamilton dynamics, which can be obtained without the use of the concept of time. Words like time, frequency, periodicity, duration, repetitivity and speed which are commonly employed in natural language to describe reality from our observer's perspective are unspeakable in physics, even if they allow to provide a satisfactory description of most common situations in which Nature is not examinated at a fundamental level.

\section{References}

[1] C. Rovelli, Quantum Gravity (Cambridge University Press, Cambridge, UK, 2006)

[2] C. Callender, Scientific American 302, 58 - 65 (2010)

[3] J.B. Barbour, Class. Quantum Gravity 11, 2875 (1994) and Class.Quantum Gravity 11, 2898 (1994)

[4] E. Prati, Journal of Physics: Conference Series 306, 012013 (2011)

[5] P Zenczykowski, J. Phys. A: Math. Theor. 42, 045204(2009)

[6] B. S. De Witt, Phys. Rev. 160, 1113 (1967)

[7] S. A. Diddams et al., Science 293, 825 (2001)

[8] P. Heavner et al., Metrologia, 42, 5, 411 (2005)

[9] W. H. Oskay et al., Phys. Rev. Lett. 97, 020801 (2006)

[10] Lindner et al., Phys. Rev. Lett. 95, 040401 (2005)

[11] A. Palacios, Phys. Rev. Lett. 103, 253001 (2009)

[12] H. Niikura, Nature 417, 917-922 (2002)

[13] H. B. v. Linden et al., Multiphonon processes Studies in Modern Optics, 8, pag. 25 (Cambridge University Press, Cambridge, UK, 2001)

[14] P. Salieres et al., Science 292, 902 (2002)

[15] P. H Sydenham and R. Thorn, Handbook of Measuring System Design, Definition of Time, Time Interval and Frequency, p. 1341 (Wiley, New York, USA, 2005)

[16] L. D. Landau, E. M. Lifshitz, Vol. 1: Mechanics (Pergamon Press, Oxford, UK, 1969) 
